\title{
La influencia del apoyo social en cuidadores de personas con deterioro cognitivo
}

\section{o demencia.}

\section{The influence of social support on caregivers of people with cognitive impairment or dementia.}

\author{
Paula Antelo *, Pablo Espinosa * \\ *Universidad de A Coruña,
}

\begin{abstract}
Resumen
El apoyo social se define como un conjunto de transacciones que implican ayuda, afecto y afirmación. Así, el objetivo de la investigación ha sido desarrollar un modelo que explique como la frecuencia de cuidados influye en la satisfacción de los cuidadores de personas con deterioro cognitivo o demencia a través de variables como el apoyo social o la sobrecarga. Los resultados obtenidos confirman que la frecuencia de cuidados predice la satisfacción. Asimismo, se demuestra que están mediados por el apoyo social y la sobrecarga, conllevando el primero a una mayor satisfacción y la sobrecarga a una disminución de la misma.

Palabras clave: apoyo social, cuidadores, deterioro cognitivo, sobrecarga
\end{abstract}

\begin{abstract}
Social support is defined as a set of transactions that involve help, affection, and affirmation. The objective of the research has been to develop a model that explains how the frequency of care influences the satisfaction of caregivers of people with cognitive impairment or dementia through such variables as social support or overload. The results confirm that the frequency of care predicts satisfaction. Likewise, it is shown that they are mediated by social support and overload, leading to the first to greater satisfaction and the overload to a decrease in it.

Key words: social support, caregivers, cognitive impairment, overloads
\end{abstract}

En los últimos años, debido incremento del envejecimiento de la población, la demencia se ha convertido en el trastorno neurodegenerativo por excelencia, así como un problema de grandes dimensiones médicas y sociales (Martínez-Alcalá, Pliego-Pastrana, López-Noguerola, Rosales-Lagarde \& Zaleta-Arias, 2015).

La demencia es un síndrome de naturaleza crónica y progresiva, caracterizada por el deterioro de las funciones cognoscitivas y de la conducta, lo que ocasiona discapacidad y dependencia. (Laks, Goren, Dueñas, Novick \& Kahle-Wrobleski, 2016). Así, en el curso de estas patologías los pacientes se vuelven dependientes de sus familiares (Shua-Haim, Haim, Shi, Kuo\& Smith, 2001).

En relación a los cuidados, prestar atención a una persona con deterioro cognitivo o demencia está asociado a una carga emocional, física y financiera. Ser cuidador familiar ha demostrado ser un factor de riesgo para la depresión, ansiedad, el aislamiento y la angustia (Warchol-Biedermann, Gregersen, Maibom, Millá, \& Maseda, 2014), (Johannessen, Bruvik, \& Hauge, 2015).

Consiguientemente, la angustia y el estrés que los cuidados provocan se asocian con problemas de salud, menor calidad de vida y satisfacción. Además, la sobrecarga del cuidador es un predictor de institucionalización anticipada (Prick, de Lange, Twisk \& Pot, 2015).

Por ello, el apoyo social y el afrontamiento han demostrado ser las dos variables moduladoras o modificadoras del estrés en el cuidador que más atención deben recibir. La existencia de las mismas aseguran que el cuidador tiene recursos para hacer frente a situaciones de estrés y malestar psicológico (Yanguas, 2000).

Por su parte, el apoyo social ha sido definido como un concepto multidimensional que indica una variedad de los recursos reales o percibidos (Cené et al., 2013), incluyendo las tres dimensiones de apoyo: emocional, tangible, instrumental o informativo, disponibles para el individuo a través de vínculos con otros (Trepte, Dienlin \& Reinecke, 2015), (Schroevers, Ranchor \& Sanderman, 2003).

Referente a las estrategias de afrontamiento pueden ser definidas como el modo en que las personas resuelven o se sobreponen a las condiciones vitales estresantes, en tanto que se podría admitir la reciprocidad entre el estrés y el afrontamiento (Chen, Huang, Yeh, Huang \& Chen., 2015).

Los métodos de afrontamiento pueden ser clasificados en dos grandes patrones: afrontamiento positivo y afrontamiento negativo. El primero implica que las personas hacen un esfuerzo activo por gestionar los factores de estrés y hacen esfuerzos para cambiar o controlar las circunstancias estresantes y gestionar las respuestas emocionales. El segundo conlleva a un 
desenganche de las circunstancias del cuidado (Lou, Lau, \& Cheung, 2015).

Por el contrario, la demanda de cuidados conduce a factores de riesgo como la sobrecarga o el aislamiento social, los cuales deben ser analizados (Mahoney, LaRose \& Mahoney (2015)

El aislamiento se refiere a la condición de no tener vínculos con los demás, pudiendo desembocar en una desconexión social del sistema (Ferreira Alves, Magalhaes, Viola \& Simoes, 2014). Consecuentemente, se ha encontrado una fuerte asociación entre el aislamiento social y la depresión, independientemente de la soledad, incluyendo además resultados negativos como la mortalidad y el deterioro cognitivo (Medvene et al., 2015).

La sobrecarga puede ser definida como un resultado de combinaciones de trabajo físico, emocional $y$ restricciones sociales, esto hace referencia a un estado psicológico que surge al cuidar (Cerquera, Granados \& Buitrago, 2012). Además, los cuidadores de personas con deterioro cognitivo se encuentran con síntomas conductuales y psicológico que causa la enfermedad. Por ello, se enfrentan a un doble desafío, el cual acaba desarrollando en muchas ocasiones síntomas de sobrecarga e indicios depresivos en el propio cuidador. (Fauth, Femia \& Zarit, 2016).

Por todo ello, es evidente que lo demanda de cuidados en personas con deterioro cognitivo provoca aislamiento y sobrecarga en los cuidadores, asociados con problemas físicos de salud, morbilidad psiquiátrica y una menor calidad de vida (Prick, de Lange, Twisk \& Pot, 2015).

Así, Kenigsberg y su equipo (2016) exponen que aunque la investigación actual es escasa, cada vez son mayores los estudios aleatorizados creados para mostrar el efecto significativo y duradero de las intervenciones psicosociales, las cuales han demostrado ser eficaces en la gestión funcional, de comportamiento y síntomas psicológicos de demencia. Sin embargo, los procesos básicos implicados deben identificarse mejor.

\section{Objetivos e hipótesis}

Por ello, el presente estudio tiene como objetivo principal desarrollar un modelo que analice la relación de la dedicación del cuidador con su nivel de satisfacción considerando variables como el apoyo social, la satisfacción, la sobrecarga y el aislamiento. En este sentido, se plantean las siguientes hipótesis:

La frecuencia de cuidado se relaciona con la satisfacción en el sentido de que a una mayor dedicación se relaciona con menos satisfacción con la vida

La sobrecarga media en la relación entre frecuencia de cuidado y satisfacción, y permite explicar el vínculo entre ambas. Una mayor frecuencia de cuidado se relaciona con más sobrecarga y a su vez, más sobrecarga se relaciona con una menor satisfacción.

El apoyo social media en la relación entre frecuencia de cuidado y satisfacción. Una menor frecuencia de cuidado se relaciona con una mayor percepción de apoyo social y el apoyo social se relaciona con una mayor satisfacción vital.

\section{Método}

\section{Participantes.}

La muestra objeto de estudio está compuesta por 40 personas. De ellos, un $30 \% \quad(n=12)$ son hombres y un $70 \%(n=28)$ son mujeres. La media de edad de la muestra fue de 60,48 (SD=13.22) años.

\section{Instrumento para la recogida de datos.}

Los datos de interés han sido obtenidos a través de un protocolo de recogida de datos que consta de una de escalas que a continuación se describen:

Cuestionario demográfico. (elaboración propia). Preguntas relevantes sobre la situación del cuidador, la frecuencia de cuidados, el apoyo con el que cuenta, etc.

Cuestionario de apoyo social funcional Duke-Unk. (Bellón, Delgado, De Dios \& Lardelli, 1996). Consta de 11 ítems medidos en una escala del 1 al 5.

Escala de amistad (Hawtorne, 2006). Consiste en 6 items medidos en una escala de 1 a 5 . Mide el aislamiento social.

Subescala de satisfacción. (Hahn, Cella, Bode \& Hanrahan, 2010). Consta de 9 items tomados del cuestionario de bienestar subjetivo más amplio de Hahn et al. Refleja el grado de satisfacción que el paciente tiene con diversos aspectos de su vida.

Escala de sobrecarga del cuidador de Zarit. (Martín, Salvado, Nadal, Mijo, Rico, Lanz \& Taussing, 1996). Mide el grado de sobrecarga subjetiva de los cuidadores de mayores afectados por trastornos mentales. Consta de 22 items.

\section{Análisis de datos.}

Se han utilizado los paquetes estadísticos SPSS y AMOS. Con ellos se ha llevado a cabo un análisis correlacional de los datos con el objetivo de desarrollar un modelo de ecuaciones estructurales para determinar que variables median en la relación entre la recepción de visitas y la satisfacción de los participantes.

\section{Resultados}

\section{Análisis de fiabilidad.}

El análisis de la fiabilidad de las escalas del cuestionario muestra una consistencia interna aceptable. Los alphas de Cronbach son: Cuestionario de Apoyo Social Duke $=.92$, Escala de Amistad $=.67$, Escala de Sobrecarga Zarit $=.90$, y finalmente; la Subescala de satisfacción $=.91$

\section{Análisis descriptivo.}

A continuación se describen las principales características de los participantes que resultaron de interés para el presente estudio.

En cuanto a la situación de convivencia el $72.5 \%$ viven con el familiar al que cuidan mientras un $25.5 \%$ residen solos. Respecto a la situación de convivencia, el $67.5 \%$ de los cuidadores están casados, el $12.5 \%$ están viudos, un $7.5 \%$ solteros y otro $7.5 \%$ divorciados, y finalmente, un $5.0 \%$ residen en pareja.

En relación al parentesco, un $50 \%$ de los personas encuestadas son cuidadores de sus madres, un $37.5 \%$ de 
su pareja, un $7.5 \%$ de su padre, un $2.5 \%$ de sus hermanos y otros $2.5 \%$ de su tío/a. A la par, atendiendo a su estatus laboral el $42,5 \%$ son trabajadores activos mientras el $40 \%$ están jubilados, el 10\% se encuentran en situación de desempleo y un 7.5\% son empleados/as del hogar.

Respecto a la fase de la enfermedad, los resultados muestran que el $72.5 \%$ de los receptores de cuidados se encuentran en la fase media de la enfermedad, mientras el $12.5 \%$ en la fase grave y un $15.0 \%$ en los inicios. Analizando el tiempo que se llevan brindando cuidados se dividen en cinco categorías: menos de un año (10\%), de 1 a 2 años (22.5\%), de 2 a 5 años (40\%), más de 5 años $(17.5 \%)$ y más de 10 años $(10 \%)$.

Por otro lado, respecto al tiempo que dedican brindando cuidados el $45.5 \%$ de la población objeto de estudio han respondido unas horas al día mientras otro $40 \%$ han afirmado que dedicar todo el tiempo al cuidado de su familiar y finalmente, un $15 \%$ de la población afirma brindarlos algunas horas a la semana.

Por último, el 100\% de los participantes afirman haber sentido ayuda y apoyo de los profesionales del centro cuando lo han necesitado. Igualmente, el total de la población afirma que la atención recibida por el equipo ha sido de gran ayuda.

Respecto a los cuestionarios relacionadas con la sobrecarga, satisfacción y apoyo social, que se midieron en una escala de 1 a 5 , encontramos que los participantes muestran un nivel medio de sobrecarga $(M=2.70$; $S D=.76$ ), un nivel considerable de apoyo social medido a través de la escala de amistad $(M=4.01 ; S D=.82)$ y de la escala de apoyo social funcional $(M=3.69 ; S D=1.05)$ y un nivel medio de satisfacción $(M=3.23 ; S D=.89)$. Entre sexos, solo encontramos diferencias significativas en la sobrecarga percibida $[t(38)=2.22, \quad p<.05$; hombres: $(M=2.31 ; S D=.69)$; mujeres: $(M=2.87 ; S D=.74)]$.

\section{Modelo de ecuaciones estructurales.}

Se desarrolló un modelo de ecuaciones estructurales para determinar las relaciones entre las variables del estudio. Este modelo no resultó significativo para los cuidadores varones, pero sí para las mujeres.

El modelo de ecuaciones estructurales desarrollado explica las relaciones entre las variables de interés para el estudio, así como la predicción de la satisfacción mediante estas (Figura 1). El ajuste del modelo es adecuado ( $R M S E A=.001)$

En primer lugar se observa que la frecuencia de cuidado por parte de las cuidadoras (una puntuación de 1 indicaba una frecuencia de cuidado muy elevada), tienen un efecto total significativo sobre niveles de satisfacción. Del mismo modo, se confirma que la satisfacción está mediada por la el apoyo social percibido, medido a través de la escala de amistad y también por la sobrecarga.

Como era previsible, se observa una relación negativa entre la frecuencia de cuidados y la sobrecarga, reflejando con ello como la dedicación continuada conlleva a la sobrecarga y consecuentemente afecta negativamente a la satisfacción del individuo.

Razonablemente, a raíz de la dedicación de cuidados se observa como el apoyo social influye positivamente en la satisfacción y, contrariamente, como la frecuencia de los mismos influyen en la sobrecarga y se vincula a unos bajos niveles de satisfacción, evidenciados en los resultados de dicho modelo.

En la tabla 1 se presentan las correlaciones bivariadas entre las variables del modelo. Salvo la relación entre sobrecarga y apoyo social, todas ellas son significativas.

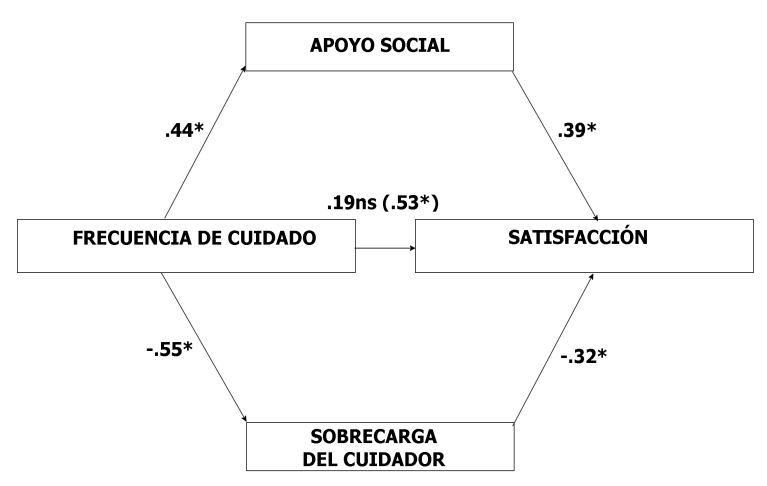

${ }^{*} \mathrm{p}<.05$; ns=no significativo. El valor entre paréntesis es el efecto total. Chi-2 del Modelo $(1, \mathrm{~N}=28)=.001, \mathrm{p}=.976 ;$ RMSEA=.001; CFI=.999; NFI=.999

Figura 1. Modelo de ecuaciones estructurales

Tabla 1.

Correlaciones bivariadas entre las variables del modelo de ecuaciones estructurales

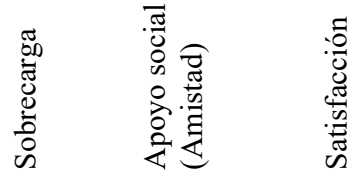

\begin{tabular}{llll}
\hline Apoyo social (Amistad) & -.22 & & \\
\hline Satisfacción & $-.51^{* *}$ & $.55^{* *}$ & \\
\hline Frecuencia de cuidados & $-.51^{* *}$ & $.44^{*}$ & $.53^{* *}$ \\
\hline
\end{tabular}

${ }^{*} p<.05,{ }^{* *} p<.01$

\section{Discusión}

El apoyo social es uno de los temas que ha generado un gran interés desde hace décadas debido al impacto positivo al que conlleva en la vida de aquellas personas que lo reciben, sobre todo, en aquellos individuos que se encuentran en circunstancias vulnerables o conflictivas de la vida.

Precisamente, es en estos momentos; los más vulnerables de la vida donde el apoyo social percibido se vuelve el principal protagonista. No obstante, la inestable situación económica y social por la que está atravesando la sociedad está conllevando a una mayor fragilidad e inestabilidad en las redes.

Por ello, dicho estudio ha pretendido acercarse a una realidad más evidente cada día y demostrar la importancia que dicho apoyo tiene en la vida de los cuidadores de personas con deterioro cognitivo o demencia.

Así, y según los resultados hallados a partir de los datos obtenidos podemos confirmar la hipótesis 1; donde se propone que la frecuencia de cuidados se relaciona 
con la satisfacción, en el sentido de que a mayor dedicación menor satisfacción con la vida. Dicho resultado se refuerza con las teorías Van Knippenbarg et al. (2017) o Trepte et al. (2015).

Del mismo modo, en relación a la hipótesis 2 , donde se plantea que "La sobrecarga media en la relación entre frecuencia de cuidado y satisfacción, y permite explicar el vínculo entre ambas. Una mayor frecuencia de cuidado se relaciona con más sobrecarga y a su vez, más sobrecarga se relaciona con menos satisfacción". Apoyan tan afirmación autores como Fauth, Femia \& Zarit (2016), Mahoney, LaRose \& Mahoney (2015), o Prick, de Lange, Twisk \& Pot, (2015).

Referente a la hipótesis 3 "El apoyo social media en la relación entre frecuencia de cuidado y satisfacción. Una menor frecuencia de cuidado se relaciona con una mayor percepción de apoyo social y el apoyo social se relaciona con una mayor satisfacción vital." los resultados la confirman. Tales deducciones concuerdan con las teorías anteriores de Zhao et al. (2014), Trepte et al. (2015) y Tuinman et al. (2006), los cuales coinciden en relacionar el apoyo social con la satisfacción en la vida.

Reforzando dicha hipótesis, diversas investigaciones investigaciones han demostrado que las personas que reciben un alto grado de apoyo social informan de una vida más satisfactoria llevando con ello a un mayor bienestar personal y social. Además, se ha verificado que dicho apoyo actúa como mediador parcial de la relación entre la autoestima y la satisfacción con la vida. Y de manera similar, la autoestima puede ser la hipótesis de mediar la asociación entre el apoyo social y la satisfacción con la vida (Zhao, Wang \& Kong, 2014), (Trepte, Dienlin \& Reinecke, 2015).

Así, el diagnóstico de deterioro cognitivo o demencia no puede ser revelado sin ofrecer intervenciones de tratamiento farmacológico y no farmacológico para el paciente y el cuidador. Diversas intervenciones no farmacológicas administradas por profesionales de la salud han mostrado efectos positivos en los pacientes de manera significativa. Del mismo modo, intervenciones educativas y psicosociales son recomendadas para los cuidadores. Así, la combinación de intervenciones de apoyo para cuidadores y medicamentos anti-colinesterasa para los pacientes parecen ser efectivas en la reducción de los síntomas depresivos y retrasar la institucionalización de los afectados. Sin embargo, la efectividad de las intervenciones para cuidadores familiares sobre la función de los pacientes en las actividades de la vida diaria ha sido poco investigada. (De Rotrou et al., 2011).

Por todo lo expuesto previamente, se incide en la construcción de programas que permitan una mayor concienciación de la población y familias sobre dicho factor; así como la generación de programas adecuados de apoyo social, siendo necesaria la generación de recursos suficientes que permitan mejorar las circunstancias de la vida que, en última instancia son responsables del deterioro. (Barrón \& Sánchez, 2001).

Consiguientemente, son cada vez mayores las investigaciones y estudios que inciden en la eficacia y beneficios de dichas intervenciones. Así, Cheng y su equipo (2017) demuestran los efectos positivos que las intervenciones basadas en la reevaluación cognitiva generan en los cuidadores, Van Knippenberg et al. (2016) confirman que la intervenciones basadas en la metodología de muestreo conllevan a emociones positivas y sentimientos de competencia en el proceso de cuidados o Rausch, Caljouw \& Van (2017), los cuales llevan a cabo una revisión sistemática con el objetivo de explorar los diferentes tipos de intervenciones existentes y los resultados que generan.

Concluyendo, se puede finalizar reflexionado el deber que tiene la sociedad actual de garantizar una buena calidad de vida a los ciudadanos, y para ello se necesitan articular instrumentos que brinden apoyo a las familias que cuidan de sus mayores dependientes de tal forma que puedan seguir desempeñando esta labor satisfactoriamente para ambas partes (Arriola \& Setién, 2004).

\section{Referencias}

Arriola, M.J. \& Setién, M.L. (2004).Cuidadores de ancianos dependientes. Demandas y estrategias de apoyo. Portularia, 4, 327-338

Barrón, A. \& Sánchez, E. (2001). Estructura social, apoyo social y salud mental. Psicothema: Revista De Psicología, 13(1), 17-23.

Bellón, J. A., Delgado, A., De Dios, J. \& Lardelli, P. (1996). Validez y fiabilidad del cuestionario de apoyo social funcional Duke-UNC-11. Atención Primaria, $18,153-163$.

Cerquera, A.R., Granados, F.J. \&Buitrago, A.M. (2012).Sobrecarga en cuidadores de pacientes con demencia tipo Alzheimer. Psychologia: avances de la disciplina, 6 (1), 35-45

Chen, H., Huang, M., Yeh, Y., Huang, W., \& Chen, C. (2015).Effectiveness of coping strategies intervention on caregiver burden among caregivers of elderly patients with dementia. Psychogeriatrics, 15(1), 20-25. doi:10.1111/psyg. 12071

Cheng, S., Mak, E. P. M., Kwok, T., Lee, D. T. F., Lam, L. C. W., \& Fung, H. (2017). Benefit-finding and effect on caregiver depression: A double-blind randomized controlled trial. Journal of Consulting and Clinical Psychology, 85(5), 521-529. doi:10.1037/ccp0000176

De Rotrou, J., Cantegreil, I., Faucounau, V., Wenisch, E., Chausson, C., Jegou, D., ... Rigaud, A. (2011). Do patients diagnosed with alzheimer's disease benefit from a psycho-educational programme for family caregivers? A randomised controlled study. International Journal of Geriatric Psychiatry, 26(8), 833-842. doi:10.1002/gps.2611

Fauth, E., Femia, E., \& Zarit, S. (2016). Resistiveness to care during assistance with activities of daily living in non-institutionalized persons with dementia: Associations with informal caregivers' stress and well-being. Aging and Mental Health, 20(9), 888-898. doi:10.1080/13607863.2015.1049114

Ferreira Alves, J., Magalhães, P., Viola, L., \& Simoes, R. (2014). Loneliness in middle and old age: Demographics, perceived health, and social satisfaction as predictors. Archives of Gerontology and 
Geriatrics,

$59(3)$

613-623.

http://dx.doi.org/10.1016/j.archger.2014.06.010

Hahn, E. A., Cella, D., Bode, R. K. \& Hanrahan, R. T. (2010). Measuring Social Well-Being in People with Chronic Illness. Social Indicators Research, 96(3), 381-401. doi: 10.1007/s11205-009-9484-Z

Hawthorne, G. (2006). Measuring social isolation in older adults: Development and initial validation of the friendship scale. Social Indicators Research, 77(3), 521-548. doi: 10.1007/s11205-005-7746-y

Johannessen, A., Bruvik, F., \&Hauge, S. (2015).Familycarers' experiences of attending a multicomponentpsychosocialintervention program for carers and persons with dementia. Journal of Multidisciplinary Healthcare, 8, 91-99. doi:10.2147/JMDH.S76093

Kenigsberg, P., Aquino, J., Berard, A., Gzil, F., Andrieu, S., Banerjee, S., Brémond, F., Buée, L., Cohen-Mansfield, J., Mangialasche, F., Platel, H., Salmon, E. \& Robert, P. (2016). Dementia beyond 2025: Knowledge and uncertainties. Dementia, 15(1), 6-21. doi:10.1177/1471301215574785

Laks, J., Goren, A., Dueñas, H., Novick, D., \& KahleWrobleski, K. (2016). Caregiving for patients with alzheimer's disease or dementia and its association with psychiatric and clinical comorbidities and other health outcomes in brazil. International Journal of Geriatric Psychiatry, 31(2), 176-185. doi:10.1002/gps.4309

Lou, V. W. Q., Lau, B., \& Cheung, K. (2015). Positive aspects of caregiving (PAC): Scale validation among chinese dementia caregivers (CG). Archives of Gerontology and Geriatrics, 60(2), 299-306. doi:10.1016/j.archger.2014.10.019

Mahoney, D. F., LaRose, S., \& Mahoney, E. L. (2015). Family caregivers' perspectives on dementia-related dressing difficulties at home: The preservation of self model. Dementia, 14(4), 494-512. doi:10.1177/1471301213501821

Martín M., Salvado, I., Nadal, S., Miji, L.C., Rico, J.M. \& Lanz, P. (1996) Adaptación para nuestro medio de la Escala Sobrecarga del Cuidador (Caregiver Burden Interview) de Zarit. Rev Gerontol, 6, 338-346

Martínez-Alcalá, C. I., Pliego-Pastrana, P., Lopez-Noguerola, J.S., Rosales-Lagarde, A., \& Zaleta-Arias, M. E. (2015). Adoption of ICT in the aging: Systematic review based on ICT for alzheimer's disease and other senile dementias. 10 th Iberian Conference on Information Systems and Technologies(pp. $1-6)$ doi:10.1109/CISTI.2015.7170393

Medvene, L., Nilsen, K., Smith, R., OfeiDodoo, S., DiLollo, A., Webster, N. \& Nance, A. (2015).Social networks and links to isolation and loneliness among elderly HCBS clients. Aging and Mental Health, , 1-9. doi:10.1080/13607863.2015.1021751

Prick, A., de Lange, J., Twisk, J., \& Pot, A. (2015). The effects of a multi-component dyadic intervention on the psychological distress of family caregivers providing care to people with dementia: A randomized controlled trial. International Psychogeriatrics, 27(12), 2031-2044. doi:10.1017/S104161021500071X
Rausch, A., Caljouw, M. A. A., \& van, d. P. (2017). Keeping the person with dementia and the informal caregiver together: A systematic review of psychosocial interventions. International Psychogeriatrics, 29(4), 583-593. doi:http://dx.doi.org/10.1017/S1041610216002106

Schroevers, M., Ranchor, A., \&Sanderman, R. (2003). The role of social support and self-esteem in the presence and course of depressive symptoms: A comparison of cancer patients and individuals from the general population. Social Science\&Medicine, 57(2), 375-385. doi:10.1016/S0277-9536(02)00366-0

Shua-Haim, J. R., Haim, T., Shi, Y., Kuo, Y. \& Smith, J. (2001).Depression among alzheimer's caregivers: Identifying risk factors. American Journal of Alzheimer's Disease and Other Dementias, 16(6), 353-359. doi:10.1177/153331750101600611

Trepte, S., Dienlin, T., \&Reinecke, L. (2015). Influence of social support received in online and offline contexts on satisfaction with social support and satisfaction with life: A longitudinal study. Media Psychology, 18(1), 74-105. doi:10.1080/15213269.2013.838904

Tuinman, M., Hoekstra, H., Fleer, J., Sleijfer, D. \& Hoekstra-Weebers, J. E. H. M. (2006). Self-esteem, social support, and mental health in survivors of testicular cancer: A comparison based on relationship status. Urologic Oncology, 24(4 SPEC. ISS.), 279-286

Van Knippenberg, Rosalia J. M., de Vugt, M. E., Ponds, R. W., Myin-Germeys, I., \& Verhey, F. R. J. (2016). Dealing with daily challenges in dementia (deal-id study): Effectiveness of the experience sampling method intervention 'Partner in sight' for spousal caregivers of people with dementia: Design of a randomized controlled trial. BMC Psychiatry, 16, 14. Retrieved

from https://search.proquest.com/docview/1791347210?acc ountid $=17197$

Warchol-Biedermann, K., Mojs, E., Gregersen, R., Maibom, K., Millán-Calenti, J.C., \& Maseda, A. (2014).What causes grief in dementia caregivers? Archives of Gerontology and Geriatrics, 59(2), 462-467. doi:10.1016/j.archger.2014.05.013

Zhao, J., Wang, Y. \& Kong, F. (2014). Exploring the mediation effect of social support and self-esteem on the relationship between humor style and life satisfaction in chinese college students. Personality and Individual Differences, 64, 126-130. doi:10.1016/j.paid.2014.02.026 